\title{
À condition d'être femme. Violette Leduc ou quand la misogynie fait écrire
}

\author{
Josée Bergeron
}

\begin{abstract}
Through Violette Leduc's autobiographical trilogy, this article shows how the exorcism of the female body and female sexuality becomes an impetus for writing. By questioning the textual figurations of self-shame, this text underlines the paradoxical tension, which ties a "feminist" writer to a phalocentric system of thought. This reflection wishes to establish the psychic and literary influence brought on by the frequentation of male worlds and to shed light on certain kinship between writing and the use of the female body. In short, the reading of Violette Leduc's texts proposed here illustrates the way that an exhibitionism of enunciation may serve as a counterpoint to the multiform experience of misogyny.
\end{abstract}

Penser l'œuvre de Violette Leduc, c'est immédiatement soulever la question de son appartenance au champ de la littérature féministe. Si son œuvre est d'abord une revendication d'existence, elle n'est jamais un cri. Il s'agit bien plus d'une plainte qui fend l'âme par l'expiation obligée d'une faute dont elle est le fruit honteux, une («mouche sur un linge blanc ${ }^{i}$ » (Leduc 1964, 56), autant qu' elle fend le corps par cette assomption difficile mais incessante de la féminité. Une brèche marque le portrait de La Bâtarde, et le texte est l'ouverture nouvelle d'où émane un râle de détresse. Violette Leduc est l'enfant non désirée, mais elle est aussi et surtout la femme non désirable qui, dans l'amour comme dans la littérature, implore: «Défriche, tu as des terres à côté de toi» (FT, 390). Violette violée dans son orgueil, fleur exposée qui s'empourpre; le seul prénom de cette femme écorchée résonne d'une souffrance singulière.

L'audace de Violette Leduc à raconter sa découverte de l'érotisme homosexuelii tout comme son admiration amoureuse pour Simone de Beauvoir ont certainement contribué à la situer dans une perspective «féministe». D'un roman à l'autre, l'histoire de Violette Leduc se répète: il s'agit d'une énonciation du corps féminin dans son désir, sa souffrance ou sa faim, tout autant qu'un plaidoyer en faveur d'une affirmation de soi libre et impudique. Voix féministe? Risquer une classification absolue s'avère inefficace dans le cas d'une auteure qui incarne à peu près toutes les formes de marginalité. Enfant naturelle d'une bonne, jeune fille solitaire, femme laide et homosexuelle, trafiquante et voleuse; Violette Leduc est en rupture avec un ordre social fondé sur une domination de la pensée masculine phallocentrique. D'ailleurs, la voix de cette écrivaine marginale s'entend d'abord comme une réponse au discours misogyne dans la mesure où celui-ci conçoit le corps féminin comme «le point de départ et le lieu d'élection de cette 
infériorisation» (Groult 22) de la femme. Avec sa trilogie autobiographique ${ }^{\mathrm{iii}}$, Violette Leduc entraîne le lecteur dans ces zones où s'active la conjuration du corps ou du sexe féminin. Notre objectif est d'en souligner ici quelques manifestations dans le rapport de Leduc à son propre visage, dans les conceptions psychiques qui résultent de son rapport aux hommes puis dans la sexualisation même de l'écriture.

\section{Le visage de la honte}

C'est d'abord sur son propre visage que Violette Leduc détecte les indices qui la mèneront à l'expérience de la misogynie. Le visage, en tant que surface perceptible et emblématique de la féminité au sens traditionnel du terme, est chez elle le premier élément d'une stigmatisation. Déjà, enfant, Violette Leduc éprouve sa «différence»: «Tu ne seras jamais comme une autre!» (LA, 22) lui reproche sa mère. Dans le regard bleu et froid de celle-ci, Violette Leduc se reconnaît «d'abord comme un sexe maudit, menacé par les mâles» (Beauvoir 15). De fait, son visage fait réapparaître celui de son père biologique, André Debaralle, «le mort, le séducteur de [sa] mère» (LB, 57). Jusqu'à la maladie pulmonaire soudaine de Violette qui ravive la honte, car elle «réincarnait André» et que «la blessure dans [sa] gorge humiliait encore [sa] mère» (LB, 59) lui rappelant sans cesse le rejet dont elle avait été l'objet.

C'est donc par l'intermédiaire de sa fille que Berthe Leduc choisit de purger son passé. Loin de nier le visage de Violette, elle s'emploie à y faire revivre les traits du fils de cette famille typique de la société patriarcale et bien-pensante. Violette, qui porte le «gros nez» et les «cheveux ternes» de son géniteur, devient pour sa mère l'indigne héritière d'un mâle tuberculeux. L'acharnement expiatoire de la mère fait ainsi du visage de Violette la preuve presque monstrueuse du lien charnel qui a uni deux univers sociaux disparates. Mais cet entêtement rageur, qui consiste à lire sur le visage de Violette la trace de l'autre qu'elle abhorre, dissimule à peine la crainte qui l'habite devant le mystère de son enfant. Aux yeux de sa mère, Violette Leduc demeure l'œuvre du secret, le réceptacle qui combine un héritage inavouable et l'image de sa propre jeunesse.

Si les textes de Violette Leduc évoquent les tentatives de sa mère pour s'approprier un pouvoir qui échappe à une femme de son époque - attitude que l'on pourrait apparenter à un certain féminisme -, ils mettent aussi en relief l'incorporation de comportements empruntés à une éthique machiste. En effet, dans La Bâtarde et La Folie en tête, Leduc montre bien que le souci de plaire et le refus de vieillir sont presque obsessionnels chez sa mère. La tyrannie du paraître, imposée par les valeurs masculines, est totalement intégrée par Berthe Leduc. Ainsi, la séduction dont elle se sert pour «réussir» bascule complètement et devient un instrument redoutable par lequel elle affirme sa supériorité et son pouvoir de domination sur sa fille. De toute évidence, un processus de «masculinisation» travaille le lien qui «unit» Violette à sa mère. En racontant une promenade parisienne avec sa mère alors qu'elle a vingt ans, Violette Leduc relate cette scène où celle-ci lui reproche: «Ne me prends pas le bras. Mais que tu es paysan!» Au-delà de la froideur qui s'en dégage, ce reproche est bien 
emblématique de leur relation. Le coup a porté, et Violette déclare: «Paysan. Le masculin m'affligeait» (LB, 133). Il faut voir dans cette anecdote bien plus qu'une allusion perfide à l'ambivalence sexuelle de Violette: en affublant sa fille d'un qualificatif masculin, Berthe Leduc parle ici au nom du mâle. Voilà bien un indice de misogynie si on admet que la suprême manifestation de la haine de la femme consiste précisément à en annihiler la féminité.

Comme nous venons de le constater, les relations de Violette avec sa mère sont empreintes d'une forme de misogynie féminine. À cette attitude de rejet, Violette Leduc répond d'abord en s'attaquant à l'institution morale. Le vol à l'étalage, qui suscite bien sûr la honte de la famille, devient pour elle le moyen de «dérober aux femmes ce qui les féminise» (LB, 179). Plus tard, l'écriture sera le moyen privilégié pour s'approprier une parole libre de contraintes et, en énonçant sa honte, pour tenter d'y échapper. Aussi, devant sa fille adulte, un sentiment étrange et ambigu semble animer Berthe Leduc, une sorte d'sangoisse face à un phénomène cette fois inconnu, qui serait son émancipation» (Bard 26). Dans une scène de La Folie en tête, l'auteur illustre combien c'est le rapport au corps qui se trouve encore au centre de la confrontation mère-fille. Au récit des malaises physiques de sa fille, Berthe Leduc, qui espère les symptômes du «retour d'âge», sourit et voit dans ce signe prématuré de la vieillesse un soulagement, une occasion pour elle de retrouver «un sexe anodin de petite fille» (FT, 235).

Par ailleurs, elle invite sa fille à «en profiter», à «prendre quelqu'un» (FT, 235). La volonté de resituer le corps de la fille - corps inutile, corps dé-féminisé puisque non-fécondé - dans le registre féminin hétérosexuel s'affirme dans un véritable crescendo. Ainsi, lorsque Violette se dit «trop moche» pour prendre quelqu'un, sa mère explose et l'invective: «Sois femme, cria-t-elle, et tu trouveras. Sois femme, bon sang» (FT, 235). Ces paroles comportent évidemment une double signification. D'une part, elles attribuent à Violette la responsabilité d'avoir échappé à son sexe; d'autre part, elles révèlent l'attitude préconisée pour une femme (du moins à l'époque) qui est justement de se «contenter» d'être femme. C'est plutôt au deuxième terme de cette explication que Violette Leduc est sensible comme en témoigne cette expression troublante que Benoîte Groult relève dans La Folie en tête: «Une femme sans homme, horreur, c'est une espèce de grand kangourou qui va partout avec une poche vide» (275).

\section{La «femme laide»}

La «femme laide»: voilà comment Simone de Beauvoir désigne Violette Leduc dans sa correspondance avec Nelson Algren. L'expression, pour le moins étonnante sous la plume de l'auteure du Deuxième sexe, confirme qu'en dépit d'un immense talent, Violette Leduc n'a jamais pu faire oublier son visage ingrat. De même, lors d'une rencontre avec l'industriel Jacques Guérin, Genet déclare: «Je veux vous faire connaître l'auteur de L'Asphyxie. C'est une femme extraordinaire. Elle est folle, laide, avare et pauvre mais elle a beaucoup de talent» (Jansiti 199). Certes, il faut dépasser ces commentaires, dont on peut douter qu'ils auraient été les mêmes au sujet d'un homme, pour constater que ce visage est réellement l'interface qui met Violette Leduc au contact de la misogynie. 
Précisons toutefois que, chez Leduc, le rapport au visage se situe dans un double registre puisqu'elle «avai[t] honte de [son] visage et qu'en même temps [elle] l'imposai[t]» (LB, 167). Une telle affirmation caractérise d'ailleurs parfaitement la parole de Violette Leduc qui oscille entre une métaphorisation de ses complexes et un exhibitionnisme de l'énonciation. D'abord, son visage témoigne de l'incapacité philosophique à sortir de soi. À ce propos, on peut considérer que le visage de Leduc expose la marginalité savamment aménagée pour elle par sa mère et dont nous parlions plus tôt. Il devient l'expression même de la honte, comme «le fait d'être rivé à soi-même, l'impossibilité radicale de se fuir pour se cacher à soi-même, la présence irrémissible du moi à soi-même» (Lévinas 113). Le sentiment d'étouffement en soi est tel qu'il entraîne chez Violette Leduc un véritable délire de persécution livré avec une sincérité troublante dans La Folie en tête.

Ensuite, par un effet spéculaire, ce visage semble refléter dans le regard de l'Autre l'impossibilité du don ou la difficulté du partage amoureux. La représentation littéraire du visage chez Leduc s'apparente à un masque rébarbatif confinant l'entreprise amoureuse à l'échec et reléguant la femme aimante dans les méandres de soi. L'obsession de la laideur, toujours présente dans cet univers littéraire, apparaît comme une sève qui nourrit les penchants masochistes de l'auteur. «Crapaud», «laideron», «potiron»: les allusions à l'apparence de son visage sont trop nombreuses pour dissimuler la peur qu'elle lit à son égard dans le regard d'autrui. C'est avec une délectation souffrante que Leduc relate ce moment où une femme lui crie: «moi, si j'avais cette tête-là, je me suiciderais» (LB, 221). Encore ici, l'insulte avoisine la misogynie dans la mesure où elle traduit le pouvoir hégémonique de la beauté érigé en valeur dominante par un système de pensée masculin ${ }^{\mathrm{iv}}$. Si la misogynie n'est pas toujours systématiquement perceptible chez Leduc, on sent bien que les constructions imaginaires d'espaces exclusivement masculins auxquels elle est confrontée sont omniprésents.

Pourtant c'est grâce à un homme, l'écrivain homosexuel Maurice Sachs, que Violette Leduc est d'abord poussée vers l'écriture. Mais si l'encouragement amical de Sachs donne le coup d'envoi à une entreprise littéraire, il faut bien dire que le projet d'écriture de Leduc s'alimente à même une forme d'ostracisme sexuel, voire de misogynie. L'œuvre, qui s'inscrit au premier plan dans le manquement affectif de la mère, révèle également un tout autre type de rapports clivés. De fait, chez Leduc, ce sont aussi les hommes qui font écrire: $\mathrm{d}^{\text {'abord un père }}{ }^{\mathrm{V}}$ qui refuse de reconnaître cette enfant bâtarde et un beau-père qui «aurait voulu [1']effacer» (LB, 56), puis «M. Pinteau» véritable figure du sexe mâle qui touche et terrorise la narratrice de L'Asphyxie ${ }^{v i}$, ensuite l'ami "Gabriel» qui épouse son «bonhomme ${ }^{\mathrm{vii}}$ ) et se dérobe à elle, puis Genet, l'écrivain homosexuel adulé, le génie intransigeant qui cultive chez Leduc une véritable "dévotion», et enfin Flavien, le jeune amant gynophobe et fuyant de La Folie en tête. Notre propos n'est pas de dresser une nomenclature des expériences misogynes qui apparaissent chez Violette Leduc, mais de voir dans quelle mesure la fréquentation de ces univers masculins agit comme véritable moteur de l'écriture. 
En plus de ses amours lesbiennes, il importe de souligner les rapports singuliers que Leduc entretient avec les hommes. En tombant systématiquement amoureuse d'homosexuels, Violette Leduc tente en quelque sorte d'inverser la valeur du principe de séduction. L'impact de sa relation troublée à son propre visage est l'un des premiers éléments qui affectent son rapport à l'autre. D'une part, Leduc s'installe dans des relations qui excluent la réciprocité; d'autre part, elle renégocie sa conception d'elle-même comme être sexué. Sa laideur, réelle ou exagérée, est alors mise à contribution pour entreprendre non seulement une négation de sa propre féminité mais aussi une désintégration d'elle-même. Sa passion pour l'homosexuel notoire Jacques Guérin - elle avoue être «malade du lui» (FT, 401) - la conduit d'ailleurs à certains excès de comportement fort éloquents. Violette Leduc échappe à la honte de sa féminité en adoptant une apparence masculine. Vêtue du «Pantalon de serge grise porté par Denis», l'ami de Guérin, et de son «pull bleu roi», elle devient «plate comme une planche à repasser» mais affiche «une décence impeccable» (FT, 401). Il en va de même avec Genet qu'elle rêve de séduire grâce à un impossible et pathétique travail de transformation: «Écrasement de mes seins. Une verge postiche» (FT, 578). Il faut sans doute voir dans l'attitude de Leduc une façon de mener comme un jeu ce mirage amoureux.

Chez Violette Leduc, le fait d'échapper aux représentations de la féminité demeure souvent la seule issue pour éviter le rejet. Ainsi, lorsqu'elle amorce une relation avec Patrice, jeune homme admiratif de son œuvre, Violette Leduc est encore une fois habitée par la crainte de décevoir. Une femme écrivaine qui a l'audace d'écrire a-t-elle le droit d'offrir un tel visage? Aussi, immédiatement après l'annonce de la visite de Patrice, Leduc est envahie d'un sentiment de panique: «Catastrophe. Mes cheveux raides, mes cheveux gras, mon nez, ma lèvre, mes champignons, mes cernes, où les cacher?» La dissimulation étant impossible, c'est par l'intégration d'un comportement masculin que Leduc échappe à la honte. Le masque du visage, trop criant, trop expressionniste, se retourne pour offrir la plus triste image du silence de l'âme et de la négation de soi. La femme «manquée», l'auteur qui exhibe sa monstruosité ne peut soutenir le regard masculin. Une volte-face s'opère lorsque Leduc désamorce la situation: «Bah, mes disgrâces s'accordent à mon paternalisme» (FT, 358). La banalisation du physique, véritable tactique de l'effacement, cède le pas au comportement le plus emblématique d'une suprématie masculine: le paternalisme. Dans la mesure où elle diffère d'une féminité prédéterminée, Violette Leduc trouve dans une forme de «désexuation» de soi la seule solution au risque de la misogynie.

\section{«Chaque mot est une passe»}

La chasse à l'autre, homme aimé et homosexuel ou figure évanescente du père, fonde le paradoxe qui stimule l'écriture de Violette Leduc. C'est pourquoi il importe finalement de questionner la tension qui innerve le texte de Leduc. Quand on songe que la puissance créatrice de l'écrivaine carbure aux rebuffades et à l'humiliation, force est de constater que son discours s'alimente à même la voix dominante de l'ostracisme et du rejet. En effet, même en reconnaissant la puissance germinative de son écriture et sa faculté à restaurer une matrice textuelle qui 
la fait renaitre, il faut admettre que Leduc y inscrit d'abord et avant tout la nature particulière de son rapport à l'autre. À ce propos, la relation entre Violette Leduc et Jacques Guérin révèle de façon microcosmique le rapport qu'elle entretient aux hommes ${ }^{\text {viii }}$ et à l'écriture. À son sujet, Violette Leduc déclare presque dans un même souffle qu'elle «l'intéresse quand [elle est] absente» (CA, 32) et qu'elle «[s']enchaîne à lui quand [elle] le hai[t]» (CA, 39). Chez Leduc, la douleur révoltée et l'imploration amoureuse ne sont jamais bien loin... Son parcours affectif et littéraire résulte bien souvent du mouvement entre ces deux pôles.

Les premières pages de $L a$ Chasse à l'amour sont tout à fait à l'image de cette tension paradoxale. Le roman s'ouvre sur un enchaînement étrange où la description presque maniaque d'une lame de rasoir, évoquant progressivement la tentation du suicide de l'auteur, sert d'amorce à une série métaphorique reliée à l'amputation. L'auteur qui «découpait [son] peignoir et [sa] chemise de nuit sur [son] dos» $(\mathrm{CA}, 18)$ est en proie à un véritable délire. Son imaginaire est mis à contribution pour traduire la douleur qui l'assaille et dont le lecteur ignore d'abord la cause. La narratrice rêve à des messieurs qu'elle «menaçai[ $t]$ avec des ciseaux» puis avoue qu'elle «visai[t] leur sexe avec son instrument étincelant» (CA, 18-19). Plus loin, elle s'imagine que quelqu'un est entré chez elle et «a coupé la toile cirée de la salle à manger» (CA, 22). La séquence presque sadomasochiste du début du roman puis celle du domicile violé préparent l'annonce d'un massacre beaucoup plus important. Quelques pages plus loin, cette explosion de révolte s'explique parce qu' «lls ont refusé le début de Ravages» (CA, 22). Ce «Ils» inclut à la fois l'éditeur, l'imprimeur, les typographes, les autres auteurs, la société entière qui, soudainement investie de la puissance mâle, «se dresse avant que [son] livre paraisse» (CA, 23) pour en violer le contenu. Même l'intervention de Simone de Beauvoir demeure inefficace pour empêcher la mutilation du texte. «Le couperet» $(\mathrm{CA}, 28)$ tombe, et Violette Leduc est laissée à son propre désastre.

Si l'auteur de Ravages compare l'amputation de son texte à «un assassinat» $(\mathrm{CA}, 22)$, c'est bien entendu parce qu'elle fait corps avec lui. Voilà d'ailleurs comment Leduc décrit sa relation fusionnelle à l'écriture: «Mon encre: du plasma; ma plume: un cordon ombilical. Mon texte dactylographié: un nouveau-né» (CA, 22). Dans La Chasse à l'amour, le rêve de vengeance précède le récit de la désolation. Tout le début du roman transcrit le revirement qui s'opère entre le silence imposé par la censure et l'expression de la révolte qui devient alors moteur de l'écriture. La plainte rauque qui émane d'un récit de vie à peine romancé constitue, pour Violette Leduc, une réplique cinglante à la rigidité de l'institution littéraire. En effet, Leduc se livre ici à un véritable cirque sordide où elle s'attaque au symbole même d'une rectitude littéraire: «le dictionnaire une merde» $(\mathrm{CA}, 20)$. Le livre, dont la mission est entre autres choses d'attribuer leur valeur aux mots, de trancher entre ce qui peut être dit de ce qu'on doit taire, devient l'exutoire de sa haine. La justice est revendiquée lorsque Leduc donne «une vingtaine de gifles» au dictionnaire et qu'elle s'adresse carrément à lui: «Si j'ai mal, tu as mal aussi!» (CA, 20). L'écriture qui fait renaître est donc ici hachée menue par la censure, cette «guillotine cachée» qui «tranche vos feuillets» $(\mathrm{CA}, 22)$. 
L'ablation des passages amoureux qui devaient originellement constituer la première partie de Ravages a été trop douloureuse pour que Violette Leduc n'en décrive pas les séquelles. Voilà sans doute pourquoi cet épisode tragique l'amène à penser que la littérature n'est plus le berceau d'une reconstruction mais un lit de parasites qui ressemblent «à des verges molles» $(\mathrm{CA}, 24)$. Ce constat amer, que Leduc présente avec violence dans son dernier livre, est déjà présent dans $L a$ Folie en tête. En relisant les dernières pages de ce roman, on comprend d'ailleurs à quel point sa volonté d'écrire est nourrie autant par un désir de plaire avoisinant la mendicité que par l'aveu ostentatoire de cette pratique honteuse. Par un rapprochement sarcastique, Violette Leduc associe la littérature à la perte de soi pouvant résulter du commerce du corps. En effet, «Écrire, c'est se prostituer» (FT, 586) et gagner son droit de dire la vérité à la sueur de son corps. L'expression pour le moins provocatrice de sa vision de l'institution littéraire a de quoi faire réfléchir. Toutefois, sa revanche ultime demeure celle d'un texte fortement sexualisé puisque c'est avec son exhibitionnisme habituel que Leduc dénonce la voix du plus fort.

En somme, le triptyque autobiographique qui superpose le récit de vie à celui du parcours littéraire de Leduc illustre bien la dynamique d'une écriture animée concurremment par la révolte et l'affaissement. Qu'il s'agisse des relations qu'elle entretient avec sa mère et avec les êtres qu'elle aime ou de son rapport à l'écriture, nous avons tenté de montrer que l'œuvre de Violette Leduc se nourrit à cette double tension. Les expériences combinées de la misogynie, de la «masculinisation», du rejet ou du silence imposé n'ont pas suffi à étouffer son souffle puissant. Dans un système où «Chaque mot est une passe» (FT, 586), Violette Leduc devait choisir: «Écrire ou se taire?» (FT, 586). Il semble bien qu'elle ait fait le bon choix.

\section{Notes}

${ }^{i}$ Nous présenterons les références aux œuvres de Violette Leduc par des abréviations suivies du numéro de page: L'Asphyxie (LA), La Bâtarde (LB), La Chasse à l'amour (CA) et La Folie en tête (FT). Ces œuvres sont parues dans la collection «L'Imaginaire» des éditions Gallimard.

ii Plusieurs pages jugées trop «sulfureuses» ont d'ailleurs été censurées et enlevées de Ravages. Ces pages d'une "sincérité intrépide» pour reprendre l'expression de Simone de Beauvoir réapparaissent dans Thérèse et Isabelle.

iii Il s'agit de La Bâtarde, La Folie en tête et La Chasse à l'amour. Soulignons que tous les textes de Leduc s'apparentent plus ou moins à l'autobiographie puisqu'elle «mêle la vérité au roman», ainsi qu'elle l'affirme dans La Bâtarde (p. 40 ).

iv À ce propos, soulignons que Violette Leduc raconte comment elle subit l'attrait de ce qu'on pourrait appeler l'entreprise de la séduction. On la retrouvera chez le chirurgien plastique, chez un coiffeur à la mode, dans les boutiques de Schiaparelli, etc. 
${ }^{v}$ La figure fantomatique du père induit d'ailleurs toute la trame autobiographique des textes.

vi Dans sa biographie de Violette Leduc, Carlo Jansiti présente L'Asphyxie comme un véritable récit de l'inceste. Il souligne que ce thème «hante l'imaginaire de Violette» (110).

vii Dans La Bâtarde, Leduc évoque ce surnom que lui donnait son futur mari, «Gabriel» (Jacques Mercier).

viii Le comportement de Violette Leduc est d'ailleurs assez similaire dans ses rapports avec Maurice Sachs et Jean Genet.

\section{Bibliographie}

\section{Euvres de Violette Leduc}

Leduc, Violette. L'Asphyxie. Paris: Gallimard, coll. «L'imaginaire», no 193, 1973 (réédition).

- La Bâtarde. Paris: Gallimard, coll. «L'imaginaire», no 351, 1964.

- La Chasse à l'amour. Paris: Gallimard, coll. «L'imaginaire», no 422, 1973.

- La Folie en tête. Paris: Gallimard, coll. «L'imaginaire», no 319, 1970.

\section{Autres}

Bard, Christine, dir. Un siècle d'antiféminisme, Paris: Fayard, 1999.

Beauvoir, Simone de. Préface de La Bâtarde, Paris: Gallimard, coll. «Limaginaire», no 351, 1964, p. 7 à 18 .

Groult, Benoîte. Cette mâle assurance, Paris: Albin Michel, 1993.

Jansiti, Carlo. Violette Leduc, Biographie, Paris: Grasset \& Fasquelle, 1999.

Lévinas, Emmanuel. De l'évasion, Paris: Librairie générale française, coll. «Biblio essais», 1982. 